\title{
Left ventricular remodeling and mitral valve surgery: Prospective study with real-time 3-dimensional echocardiography and speckle tracking
}

\author{
Dimosthenis Pandis, MD, MSc, ${ }^{a}$ Julia Grapsa, MD, ${ }^{a}$ Thanos Athanasiou, MD, PhD, FECTS, ${ }^{\text {b,c }}$ \\ Prakash Punjabi, MBBS, MChir, FRCS, ${ }^{\mathrm{a}, \mathrm{b}}$ and Petros Nihoyannopoulos, MD, FRCP, FESC ${ }^{\mathrm{a}}$
}

\begin{abstract}
Objectives: The aim of the present study was the assessment of left ventricular (LV) remodeling during the surgical management of degenerative mitral valve regurgitation (DMR) with the application of 2 emerging imaging modalities: real-time 3-dimensional echocardiography (3DE) and speckle tracking.
\end{abstract}

\begin{abstract}
Methods: A total of 40 patients with DMR were prospectively recruited. Two-dimensional echocardiography, 3DE, and speckle tracking studies were performed before surgery and 6 months postoperatively. The LV volumes, stroke volume, and ejection fraction were calculated. The strain of all myocardial segments, as well as dyssynchrony, was assessed. Receiver operating characteristic curves and regression analysis were used to assess the independent predictors of mitral regurgitation recurrence and LV remodeling.
\end{abstract}

Results: Of the 40 patients, 21 underwent mitral valve repair and 19 valve replacement. Their mean age was 59.5 \pm 15.4 years. The postoperative LV 3DE analysis revealed a significant reduction in LV end-diastolic volume $\left(103.9 \mathrm{~cm}^{3}\right.$ from $165.2 \mathrm{~cm}^{3}$ preoperatively, $\left.P<.001\right)$. A synchronous decrease in the LV end-diastolic diameter (48 $\mathrm{mm}$ from $53.5 \mathrm{~mm}$ preoperatively, $P<.001)$ and left atrial diameter $(42.8 \mathrm{~mm}$ from $53.1 \mathrm{~mm}$ preoperatively, $P=.005)$ were identified after DMR surgery. Regression analysis demonstrated incremental favor toward mitral valve repair in reverse ventricular remodeling. Independent factors were identified for regurgitation recurrence after surgery. The most significant were late diastolic $\left(\mathrm{A}^{\prime}\right)$ wave of the septal apical wall of $<3.2 \mathrm{~cm} / \mathrm{s}$ (area under the curve [AUC], 0.82), late diastolic $\left(\mathrm{A}^{\prime}\right)$ wave of the mid-septal wall of $\leq 3.66 \mathrm{~cm} / \mathrm{s}$ (AUC, 0.82), mid-lateral radial strain of $\leq-27$ (AUC, 0.81), and apical lateral radial strain of $\leq-25$ (AUC, 0.80).

Conclusions: 3DE and speckle tracking proved to be important imaging tools in assessing reverse LV remodeling after DMR surgery. Subtle regional preoperative changes in diastolic function of the septal and lateral wall could be identified preoperatively, aiding in optimizing the referral timing and recognizing potential culprits as indicators of disease recurrence after mitral repair. (J Thorac Cardiovasc Surg 2011;142:641-9)

Degenerative mitral valve regurgitation (DMR) represents a progressive dynamic entity, extending from the absence of symptoms, with subtle atrioventricular structural alterations, to end-stage cardiac failure, with irreversible myocardial changes. The process of chronic volume loading leads to the inevitable enactment of Laplace's law, by developing an increased surface of left ventricular (LV) wall area to reduce regional wall stress. The result is a gradual remodeling of the ventricular chamber (assuming a spherical shape) with the development of thin, elongated myocytes and eccentric

\footnotetext{
From the Department of Cardiovascular Sciences ${ }^{\mathrm{a}}$ and Department of Cardiothoracic

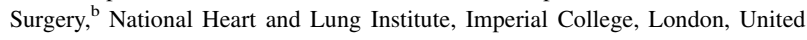
Kingdom; and Department of Biosurgery and Surgical Technology, ${ }^{\mathrm{c}}$ Imperial College London, St Mary's Hospital, London, United Kingdom.

Disclosures: Authors have nothing to disclose with regard to commercial support.

Received for publication Aug 3, 2010; revisions received Nov 4, 2010; accepted for publication Nov 20, 2010; available ahead of print Jan 31, 2011.

Address for reprints: Thanos Athanasiou, MD, PhD, FETCS, Department of Biosurgery and Surgical Technology, 10th Floor, QEQM Wing, St Mary's Hospital, London W2 1NY United Kingdom (E-mail: t.athanasiou@imperial.ac.uk). $0022-5223 / \$ 36.00$

Copyright (C) 2011 by The American Association for Thoracic Surgery doi:10.1016/j.jtcvs.2010.11.030
}

hypertrophy, adapted to the increased work $^{1}$ to maintain cardiac output. ${ }^{2,3}$

The progression to symptomatic myocardial decompensation can last for years, and clear guidelines for the classification, management, and surgical referral have been in place ${ }^{4,5}$ to help physicians and surgeons alike in decision making. Surgical referral has generally been determined by the grading the jet of mitral regurgitation, ${ }^{6,7}$ in conjunction with a visual estimation of the ventricular contractile reserve, expressed as the ejection fraction (EF).

Although traditional 2-dimensional echocardiography (2DE) has been widely used for disease classification and preoperative evaluation, it has weaknesses that adversely affect the accuracy of the LV remodeling assessment. 3DE is the current imaging modality of choice for serial evaluation of performance and shape. The novel imaging modality of $3 \mathrm{DE}$ has been developed to overcome the weaknesses of 2D acquisition and measurements and to provide more accurate and reproducible studies of cardiac structure and function. ${ }^{8-10}$

Speckle tracking (ST) is another novel imaging modality, supplementary to 3DE, for the assessment of LV global and regional function. ${ }^{11}$ It is less load-dependent and offers the 


$$
\begin{aligned}
& \text { Abbreviations and Acronyms } \\
& \text { AUC }=\text { area under the curve } \\
& \text { DMR }=\text { degenerative mitral valve regurgitation } \\
& \text { EF }=\text { ejection fraction } \\
& \text { LV }=\text { left ventricular } \\
& \text { NYHA }=\text { New York Heart Association } \\
& \text { ST }=\text { speckle tracking } \\
& \text { 3DE }=\text { real-time 3-dimensional } \\
& \text { TDI } \quad \text { echocardiography } \\
& \text { 2DE }=\text { tissue Doppler imaging }
\end{aligned}
$$

advantage of assessing the myocardial deformation independently of the angle and rotation. ${ }^{11}$ The speckle pattern can be used to track myocardial motion. ${ }^{12}$ It resembles m-mode because the speckles follow the myocardial motion. Thus, defining a region (kernel) in 1 frame, this kernel can be identified as a region in the next frame with the same size and shape with the most similar speckle pattern, and the motion of the kernel can be tracked from frame to frame. From this accumulation of regions, the algorithm can be derived. ST has been validated by ultrasonomicrometry in the longitudinal direction, as well as for rotation.

First, the pathophysiologic effects of ventricular stress in degenerative mitral valve disease can be identified preoperatively and, second, can be altered to a significant degree postoperatively because the type of surgical intervention can have differential effects on the distribution of $\mathrm{LV}$ stress. ${ }^{13}$ This particular aspect has been investigated in the present study. We have also quantified LV reverse remodeling after DMR surgery using 3DE and ST, and we have attempted to elicit whether 3DE can yield information pertaining to the possible factors of disease recurrence after mitral valve repair.

\section{MATERIALS AND METHODS}

\section{Study Population and Sample Size Calculation}

A total of 40 patients with DMR who had been referred for surgery were prospectively recruited and provided informed consent for participation in the present study. A group of 20 healthy volunteers was used as the control group, and their values were compared with those from the DMR group.

The patients were selected according to the current guidelines for surgery of mitral valve disease. ${ }^{14}$ Patients with cardiomyopathy and/or concomitant coronary artery disease were excluded from the present study. In addition, we excluded patients with heart block (second or third degree) and with fast atrial fibrillation ( $>100$ beats/min). We did not exclude patients with slow atrial fibrillation; however, care was taken for 3 different sequences to be used for 3DE and ST assessment. The images were acquired for 2 seconds or 2 heart beats. The mean value of the 3 different sequences was calculated. ${ }^{15}$ All the patients included in the present study underwent intraoperative transesophageal echocardiography, confirming successful mitral valve repair or mitral valve replacement without any residual mitral regurgitation.
The sample size was calculated as 40 patients to achieve $90 \%$ power with a significance of type I error of $\alpha=0.01$ to detect a significant difference between the 2 groups with regard to the end-diastolic ventricular remodeling parameters (before and after mitral valve surgery). All patients underwent preoperative right and left catheterization as a part of their surgical workup. The New York Heart Association (NYHA) functional class was assessed according to the clinical presentation, and only patients requiring surgery for mitral valve disease were recruited for the present study.

The local ethics committee approved the study (08/H0707/144), and the subjects gave written informed consent. All authors have read and agreed to the report as written.

\section{Echocardiography}

The echocardiographic protocol consisted of conventional transthoracic 2DE, 3DE, and ST. All examinations were performed by a single operator (J.G.). We did not determine the intra- or interobserver reproducibility, because this was previously reported by our group. ${ }^{16}$ 3DE were obtained using the GE Vivid 7 scanner (Horton, Norway) equipped with a central $\times 4$ transducer. The images were acquired from the apical 4-chamber views with the patient in the left decubitus position during a breath hold of 7 seconds. The images were then transferred to an offline workstation (4D Analysis; TomTec, Munich, Germany). Serial short-axis reconstructions of the LV volumetric data sets were then obtained, and the endocardial contour was traced at 7-mm intervals with cross-reference to the longaxis images for identification of the mitral annulus. The end-diastolic and end-systolic LV volumes and EF were calculated offline using the method of summation of discs and semiautomated border detection. The systolic volume was calculated by the subtraction of the end-systolic volume (ESV) from the end-diastolic volume (EDV), and the EF was calculated as the EDV $-\mathrm{ESV} / \mathrm{EDV}^{8-10,16}$

For the ST analysis standard, gray scale 2D images were acquired in the 2- and 4-chamber apical views, as well as the parasternal short-axis views at the level of the papillary muscles. ${ }^{11-13}$ The frame rate was 30 to 60 frames/s. The following parameters were obtained and/or calculated:

2DE: LV end-diastolic diameter, end-systolic diameter, intraventricular septal diameter and posterior wall diameter, fractional shortening and left atrial diameter, and Doppler measurements with pulsed and continuous wave

3DE: LV end-diastolic, end-systolic, and stroke volumes and ejection fraction

ST: Longitudinal, radial, and transverse strain and strain rate of LV, deformation indexes with tissue Doppler imaging (TDI) of all segments: $\mathrm{S}, \mathrm{E}^{\prime}$, and $\mathrm{A}^{\prime}$ waves (Figure 1).

The preoperative investigation was performed within 24 hours before surgery on the preoperative admission of the patient. The echocardiographic assessment was repeated 6 months after surgery using the same protocol. The definition of significant mitral regurgitation postoperatively was a mitral regurgitant volume greater than $40 \mathrm{~cm}^{3}$ (identifying more than moderate in degree regurgitation). The mean interval to the postoperative assessment was $184 \pm 17$ days.

\section{Surgery}

All patients underwent a preoperative analysis of the valvular pathologic features using conventional 2DE. Mitral regurgitation was quantified using validated 2D and Doppler methods, including the effective regurgitant orifice area, proximal flow convergence method, vena contracta, and jet area/atrium area. The grade of regurgitation was assessed using a standardized scale from 0 (none) to 4 (severe). Prolapsing or flailing leaflets were assessed according to standard criteria and Carpentier's functional classification with a precise characterization of the involved scallops or segments. ${ }^{14}$ Patients provided informed consent for both mitral valve repair and replacement. Although the former was attempted in all cases, the native valve was replaced in 19 


\section{Strain idea}
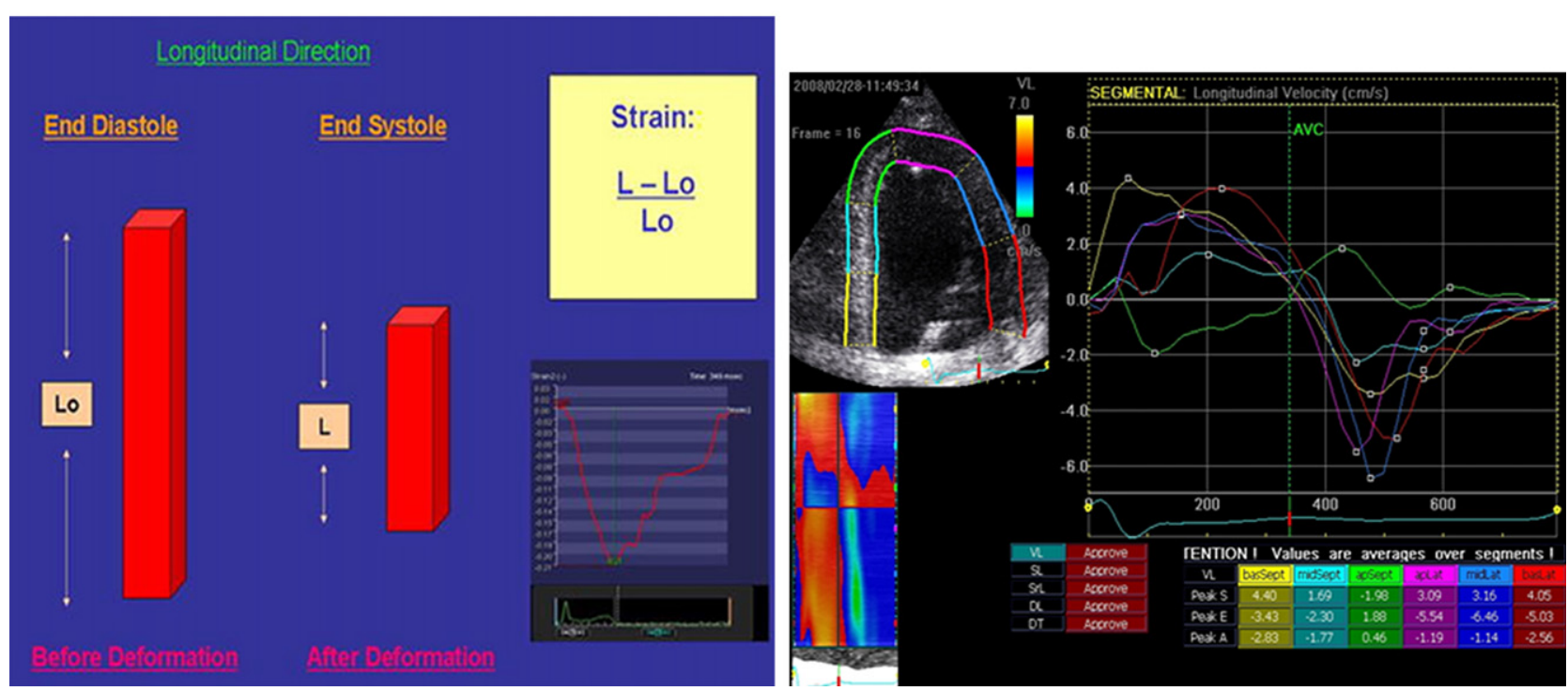

FIGURE 1. Speckle tracking $(S T)$ assessment of left ventricle postoperatively with calculation of tissue Doppler imaging (TDI) velocities and mechanism of strain.

of the 40 patients because of extensive annular calcification or extensive leaflet disease that did not allow for adequate disease-free tissue to repair the valve. All procedures were performed by the same surgeon to eliminate bias. The bileaflet chordal preservation technique was used for all 19 patients who underwent mitral valve replacement.

Mitral valve repair was accompanied by flexible annuloplasty ring insertion. With the help of the intraoperative echocardiogram, we calculated the size of the anterior leaflet and determined the optimal ring/prosthetic valve size. Atrial fibrillation was ablated with radiofrequency combined with atrial appendage ligation. Immediately after the mitral valve surgery, intraoperative transesophageal echocardiography was performed, and all patients had had a successful operation without residual regurgitation. Of the 40 patients, 21 underwent mitral valve repair: 4 patients underwent complete ring placement (CG-Future Medtronic, $27 \mathrm{~mm}$ in 2 patients and $29 \mathrm{~mm}$ in 2 patients), and 17 patients underwent band insertion (Duran band, Medtronic, in 12 patients, 5 with $25 \mathrm{~mm}, 5$ with $29 \mathrm{~mm}$, and 2 with 31 $\mathrm{mm}$; and Cosgrove Band, Edwards, in 5 patients, 3 with $27 \mathrm{~mm}$ and 2 with $29 \mathrm{~mm}$ ). The Alfieri technique was not used.

\section{Statistical Analysis}

The data are expressed as the mean \pm standard deviation for normally distributed values and the median \pm interquartile change when the variables were non-normally distributed. The normal distribution of each variable was assessed using the Kolmogorov-Smirnov test. For the non-normally distributed variables, the comparison of groups was performed using nonparametric tests, and the cutoff value for significance was $P=.05$.

The sensitivity and specificity of the cutoff points for the measured echocardiographic preoperative values were assessed to predict the postoperative recurrence of mitral regurgitation using receiver operating characteristic curves. ${ }^{17}$ The optimal cutoff value for each parameter was defined as the value giving the largest area under the curve (AUC).

Univariate and multivariate regression analyses were performed to evaluate the effect of mitral valve repair on LV remodeling and to identify the predictors of recurrent mitral regurgitation. We assessed its independent effect after adjustment for the preoperative values of the relevant LV parameters.
Statistical analyses were performed using the Statistical Package for Social Sciences, version 17.0 (SPSS, Chicago, Ill), and Medcalc, version 11.1 (Medcalc; Software bvba, Mariakerke, Belgium), software.

\section{RESULTS}

\section{Demographic Data}

The patient demographics, comorbidities, and operative data are presented in Table 1 and Figures 1 and 2. The mean patient age was $59.5 \pm 15.4$ years. Of the 40 patients, $25(62.5 \%)$ were in slow atrial fibrillation, with a mean heart rate of $76 \pm 12.7$ beats/min. At 12 hours after surgery, only 2 patients $(5 \%)$ were in atrial fibrillation and the rest were in sinus rhythm. Within 6 months of followup, $19(47.5 \%)$ of the 40 patients were in sinus rhythm.

\section{Surgical Data}

Most patients had posterior mitral valve leaflet prolapse, involving 1 or 2 scallops $(n=23,57.5 \%)$; 6 patients $(15 \%)$ had bileaflet prolapse (including the anterior and posterior leaflets), and 8 patients $(2 \%)$ were diagnosed with native valve infective endocarditis and acute severe mitral regurgitation (Table 1). For the repair group $(n=21)$, the redundant tissue was excised using triangular or quadrangular resection, and neochordae were inserted to allow for improved coaptation line and to minimize billowing. In all cases, the mitral annulus was reinforced with a flexible ring, making allowances for geometric preservation. In the replacement group $(\mathrm{n}=19)$, a bioprosthesis was chosen in 8 cases $(42 \%)$ according to the patients' age and personal preference, and $11(58 \%)$ received a mechanical valve, mostly because of young age. Antegrade cold blood 
TABLE 1. Patient characteristics

\begin{tabular}{lc}
\hline \multicolumn{1}{c}{ Demographics } & Mean \pm SD \\
\hline Age (y) & $59.5 \pm 15.4$ \\
BSA $\left(\mathrm{m}^{2}\right)$ & $1.6 \pm 0.3$ \\
BPT (min) & $97 \pm 23.77$ \\
CCT (min) & $65.9 \pm 21.8$ \\
EuroScore & $5.4 \pm 2.6$ \\
Mechanism of regurgitation & \\
AMVLP & 0 \\
PMVLP & 23 \\
Mixed prolapse & 6 \\
Annular dilation & 3 \\
Endocarditis & 8 \\
NYHA class & \\
I & 1 \\
II & 10 \\
III & 22 \\
IV & 7 \\
Procedure & \\
Repair & 21 \\
Replacement & 19 \\
\hline
\end{tabular}

$B S A$, Body surface area; $B P T$, bypass time; $C C T$, cross-clamp time; $A M V P$, anterior mitral valve leaflet prolapse; $P M V P$, posterior mitral valve leaflet prolapse; $N Y H A$, New York Heart Association.

cardioplegia was used in all cases. The mean cross-clamp and bypass times are listed in Table 1 .

\section{Preoperative Echocardiographic Data}

The LV end-diastolic diameter was $53.5 \pm 14.8 \mathrm{~mm}$ (range, 43-64 mm), and the LV end-systolic diameter was $33 \pm 12 \mathrm{~mm}$ (range, 24-49 $\mathrm{mm}$ ). The fractional shortening of the left ventricle was $33.5 \% \pm 7.8 \%$ (range, $16 \%-$ $38 \%$ ). The interventricular wall had a mean thickness of $9 \pm 1 \mathrm{~mm}$ (range, $7-12 \mathrm{~mm}$ ) and the posterior wall of 9 $\pm 0.9 \mathrm{~mm}$ (range, $7-11 \mathrm{~mm}$ ). The left atrium was dilated $(53.1 \pm 12 \mathrm{~mm})$. The mitral regurgitant volume (using the proximal flow convergence method) was $88 \pm 13.5 \mathrm{~cm}^{3}$ (range, 67-108 $\mathrm{cm}^{3}$ ). The mean effective regurgitant orifice area preoperatively was $0.46 \pm 0.09 \mathrm{~cm}^{2}$.

The 3DE studies showed the LV EDV was $165.2 \pm$ $50.4 \mathrm{~cm}^{3}$ (range, $89.1-354.7 \mathrm{~cm}^{3}$ ), and the LV ESV was $65.2 \pm 23.9 \mathrm{~cm}^{3}$ (range, 23.4-123.8 $\mathrm{cm}^{3}$ ). The mean $\mathrm{LV}$ stroke volume was $85 \pm 42.6 \mathrm{~cm}^{3}$ (range, 55.6$286.8 \mathrm{~cm}^{3}$ ). As a result, the LVEF was $65.3 \% \pm 9.3 \%$ (range, 38.6\%-80.8\%).

The global LV radial strain was $-25 \pm-5.7 \mathrm{~mm}$ (range, -34 to $-29 \mathrm{~mm}$ ). The global $\mathrm{LV}$ radial strain rate was $1.1 \% \pm 0.4 \%$ (range, $0.1 \%-2.8 \%$ ). The longitudinal LV strain was $18 \pm 5 \mathrm{~mm}$ (range, 9-36 mm), and longitudinal strain rate was $1.1 \% \pm 0.3 \%$ (range, $0.5 \%-2.3 \%$ ). All myocardial segments were assessed using TDI: the mean $\mathrm{S}$ wave was $4 \pm 2.2 \mathrm{~m} / \mathrm{s}$, mean $\mathrm{E}^{\prime}$ wave was $4.1 \pm 2.3$ $\mathrm{cm} / \mathrm{s}$, and mean $\mathrm{A}^{\prime}$ wave was $3.7 \pm 2.5 \mathrm{~cm} / \mathrm{s}$.

\section{Postoperative Values}

The fractional shortening postoperatively was $40 \% \pm$ $10.2 \%$, similar to the preoperative value $(P=.62)$. The intraventricular septum thickness was $9 \pm 0.9 \mathrm{~mm}$, similar to the preoperative value $(P=.8)$. The results for the posterior wall were also similar with a postoperative thickness of 9.8 $\pm 1.3 \mathrm{~mm}(P=.42)$.

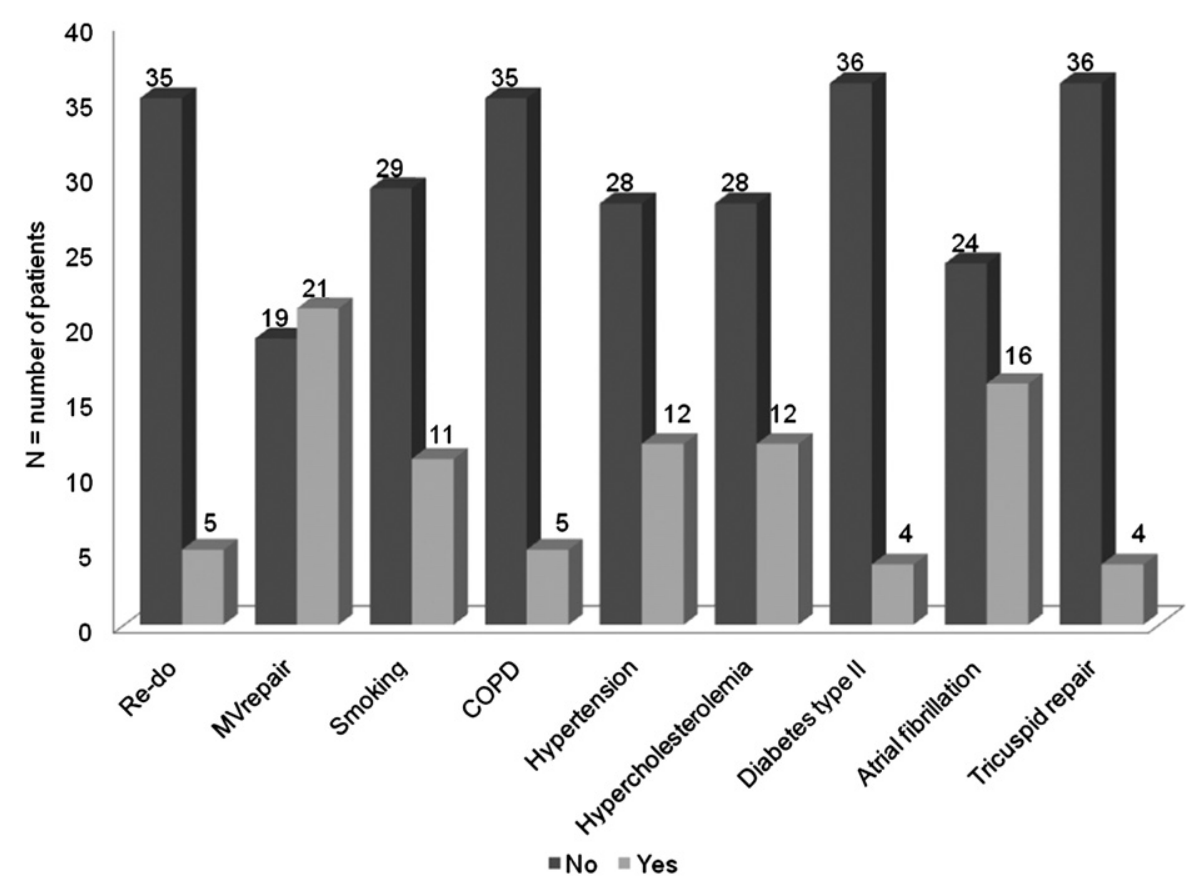

FIGURE 2. Graphic representation of prevalence of comorbidities within study group. REDO, Reoperation at study; $C O P D$, chronic obstructive pulmonary disease. 
TABLE 2. Left ventricular changes after mitral valve surgery

\begin{tabular}{lccr}
\hline \multicolumn{1}{c}{ Variable } & Preoperatively & Postoperatively & $\boldsymbol{P}$ value \\
\hline LVED $(\mathrm{mm})$ & $53.5 \pm 14.8$ & $48 \pm 8.2$ & $<.001$ \\
LV EDV $\left(\mathrm{cm}^{3}\right)$ & $165.2 \pm 50.4$ & $103.9 \pm 34.2$ & $<.001$ \\
LA $(\mathrm{mm})$ & $53.1 \pm 13$ & $42.8 \pm 16$ & .005 \\
\hline
\end{tabular}

Data presented as mean $\pm \mathrm{SD}$. $L V E D$, Left ventricular end-diastolic diameter; $L V E D V$, left ventricular end-diastolic volume; $L A$, left atrial diameter.

2DE demonstrated a LV end-diastolic diameter of $48 \pm$ $8.2 \mathrm{~mm}$ (range, $39-56 \mathrm{~mm} ; P<.001$ compared with preoperatively) and an LV end-systolic diameter of $26 \pm 5.4 \mathrm{~mm}$ (range, $18-40 \mathrm{~mm}$ ), lower than the size preoperatively $(P<.05$; Table 2$)$.

3DE demonstrated that the LV EDV was significantly lower than the EDV preoperatively: $103.9 \pm 34.2 \mathrm{~cm}^{3}$ (range, $\left.62-285.3 \mathrm{~cm}^{3} ; P<.001\right)$. The LV end-systolic volume was also reduced compared with the preoperative volume: 43.1 $\pm 12.6 \mathrm{~cm}^{3}$ (range, $21.1-104.6 \mathrm{~cm}^{3} ; P=.032$ ). Furthermore, when multivariate regression analysis was performed with the dependent variables LV EDV and ESV, taking into account the preoperative values and surgical treatment type (repair vs replacement). It was demonstrated that the LV EDV improved to a different degree in the repair group (from 63.2 to $22 \mathrm{~cm}^{3}$ ) than in the replacement group (from 67.8 to $54.7 \mathrm{~cm}^{3}$; mitral valve repair $=1$, beta coefficient $=11.21$, standard error $=5.41, P=.04)$. Similarly, a trend was identified for the ESV (mitral valve repair $=1$, beta coefficient $=16.73$, standard error $=9.45$, $P=.08$ ). We also assessed the effect of postoperative atrial fibrillation and the size of the ring on the EDV and ESV and did not identified any statistically significant difference. The LV stroke volume was $50.8 \pm 9.3 \mathrm{~cm}^{3}$ (range, $41-59 \mathrm{~cm}^{3}$ ), significantly lower than the stroke volume preoperatively $(P<.05)$. The LVEF was significantly lower overall $(42 \% \pm 7.6 \%$; range, $39.7 \%-58 \%$; $P<.01)$.

The LV global strain was measured and compared with the strain preoperatively (Figure 3 ). The global LV radial strain was increased $(-18 \pm-7.3 \mathrm{~mm}$; range, -5 to -49 $\mathrm{mm} ; P<.01)$. The global $\mathrm{LV}$ radial strain rate was $1.3 \%$ $\pm 0.5 \%$ (range, $0 \%-2.8 \% ; P<.01$ ). The longitudinal LV strain was $12 \pm 7.4 \mathrm{~mm}$ (range, $2-32 \mathrm{~mm} ; P<.01$ ), and the longitudinal strain rate was decreased $(0.9 \% \pm 0.2 \%$; range, $0 \%-1.5 \% ; P<.01$; Table 3 ). Finally, the TDI values were similar to the values preoperatively (mean $\mathrm{S}$ wave: 4.9 $\pm 3.5 \mathrm{~cm} / \mathrm{s}$; range, $0.4-15.1 \mathrm{~cm} / \mathrm{s} ; P=.18$; $\mathrm{E}^{\prime}$ wave: $4.3 \pm$ $3.7 \mathrm{~cm} / \mathrm{s} ;$ range, $0.9-16 \mathrm{~cm} / \mathrm{s} ; P=.2$; and $\mathrm{A}^{\prime}$ wave: $4.7 \pm$ $3.7 \mathrm{~cm} / \mathrm{s}$, range, $0.4-15.8 \mathrm{~cm} / \mathrm{s} ; P=.12$ ).

\section{Differences in LV Remodeling in Patients With Recurrent Mitral Regurgitation}

Five patients $(23.8 \%)$ from the repair group developed recurrent mitral regurgitation within 6 months after surgery, with no relation to intraoperative factors. Three patients developed moderate mitral regurgitation $\left(40-60 \mathrm{~cm}^{3}\right)$ and 2 patients developed moderate to severe mitral regurgitation $\left(>60 \mathrm{~cm}^{3}\right)$. Of these patients with recurrent mitral regurgitation, 2 had functional NYHA class II, 2 had functional class III, and only 1 had NYHA class IV. A total of 31 patients with successful mitral valve surgery had NYHA class I and 4 NYHA class II. When NYHA was included in the regression analysis, the NYHA class proved to be nonsignificant factor in determining the development of recurrent mitral regurgitation $(P=.43)$. Similarly, age $(P=.31)$, gen$\operatorname{der}(P=.51)$, preoperative atrial fibrillation $(P=.86)$, postoperative atrial fibrillation $(P=0.94)$, ring size used $(P=.14)$, and posterior leaflet repair $(P=.18)$ were not predictors of mitral regurgitation on univariate logistic regression analysis.

The patients who did not develop mitral regurgitation postoperatively (group A) had a LV EDV similar to that of the patients who developed recurrent mitral regurgitation (group B). The preoperative data were $176.3 \pm 79.7 \mathrm{~cm}^{3}$ for group A and $147.4 \pm 37.7 \mathrm{~cm}^{3}$ for group B $(P=.46)$, and the postoperative data were $174.9 \pm 110 \mathrm{~cm}^{3}$ for group A and $130.7 \pm 37.1 \mathrm{~cm}^{3}$ for group $\mathrm{B} ; P=.48$. However, a significant difference was found in the preoperative LVEF between the 2 groups (preoperative data: $71.2 \% \pm 7.8 \%$ for group $\mathrm{A}$ and $58.5 \% \pm 11.9 \%$ for group $\mathrm{B}, P=.03$ ), no postoperative difference was found $(P=.21)$. In addition, no significant difference was found in the LV ESV or LV stroke volume pre- and postoperatively between the 2 groups (preoperatively 0.37 and 0.2 vs postoperatively 0.31 and 0.54 , respectively).

The findings showed that when the septal apical wall and the mid-apical wall had decreased TDI velocities in late diastole ( $\mathrm{A}^{\prime}$ wave) preoperatively $(<3.2 \mathrm{~cm} / \mathrm{s}$; AUC, 0.823 ; and $\leq 3.66 \mathrm{~cm} / \mathrm{s}$; AUC, 0.815 , respectively), the possibility of recurrent regurgitation was high.

Additionally, when the mid-lateral wall exhibited decreased systolic TDI velocities ( $\mathrm{S}<4.02 \mathrm{~cm} / \mathrm{s}$ ) and, hence, systolic dysfunction, disease recurrence was likely (AUC, 0.738). Preoperative diastolic dysfunction of the basal and mid-septal segments ( $\mathrm{A}^{\prime}$ wave) was also identified as a strong predictor of disease recurrence after mitral valve repair $\left(\mathrm{A}^{\prime}>3.87\right.$ $\mathrm{cm} / \mathrm{s} ;$ AUC, $0.708 ; \mathrm{A}^{\prime}>5.14 \mathrm{~cm} / \mathrm{s} ;$ AUC 0.708 , respectively). ST identified the strongest predictors of mitral valve repair failure, when interrogating the left lateral wall preoperative deformation. When the radial strain of the apical and midlateral wall exhibited decreased radial strain preoperatively ( $\leq-25$ and $\leq-27$, respectively), mitral valvular regurgitation was very likely to recur, despite optimal repair (AUC, 0.80 and 0.814 , respectively). When considering the velocities and volumes, the preoperative mitral valvular peak pressure gradient $<94.1 \mathrm{~mm} \mathrm{Hg}$ (AUC, 0.736 ) and peak mitral velocity $\leq 4.85 \mathrm{~mm} / \mathrm{s}$ (AUC, 0.736 ) were strong indicators of mitral valve repair failure. We have presented the findings hierarchically in Table 4. 

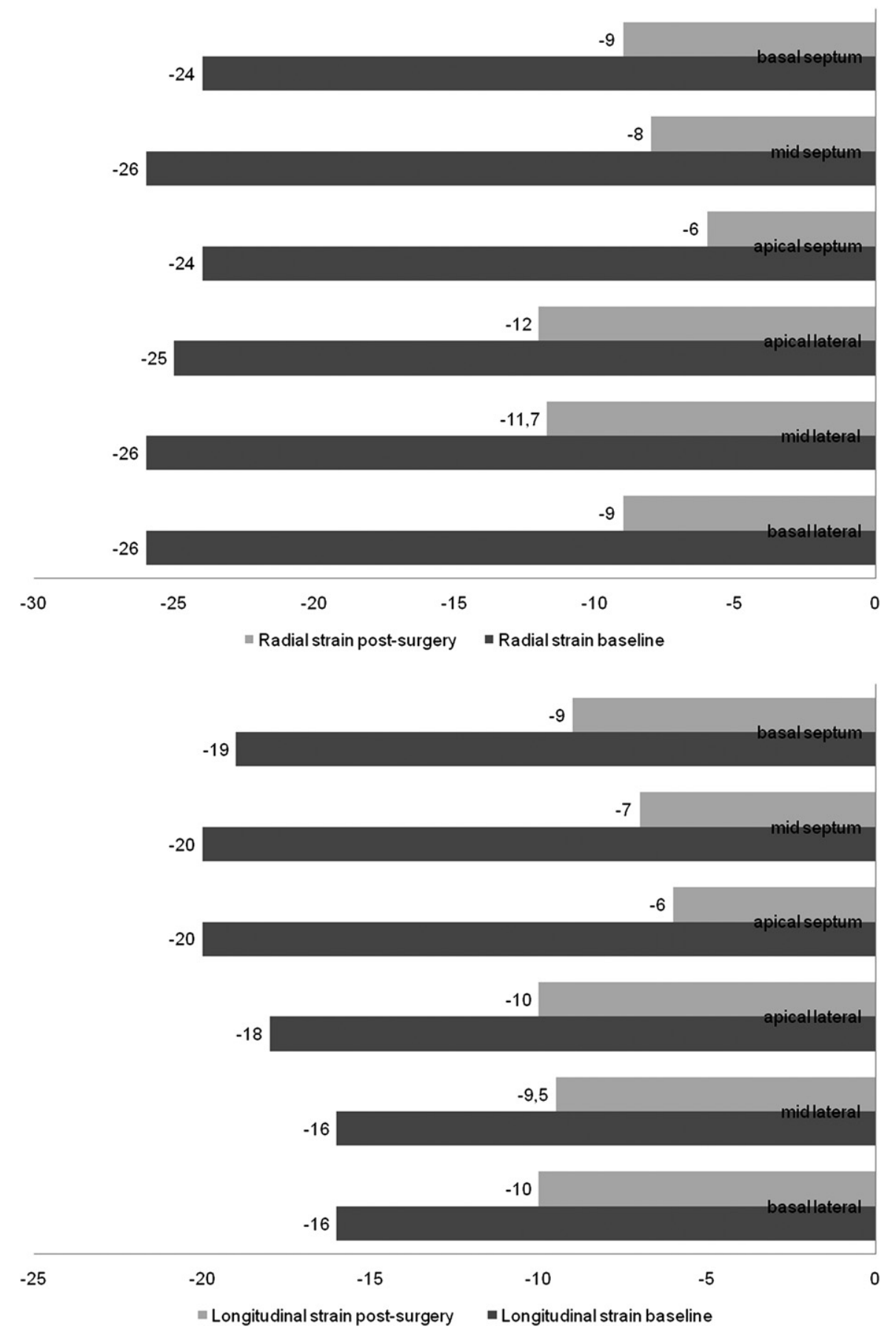

FIGURE 3. Speckle tracking ( $S T)$ results for radial and longitudinal left ventricular $(L V)$ strain.

\section{Comparison Between Repair and Replacement}

The differential effect of mitral valve repair on the postoperative LV (after adjustment for the preoperative values) EDV and ESV was described earlier. Furthermore, the LVEF preoperatively was similar between the 2 groups (replacement group, $62.3 \% \pm 13.3 \%$ vs repair, $58.1 \% \pm 10.8 \%$, $P=.22$ ) as well as postoperatively (replacement, $55.3 \% \pm$ $9.3 \%$; repair, $61 \% \pm 7.5 \% ; P=.14)$. The LV ESV pre and postoperatively was similar within the 2 groups (replacement, $58.5 \pm 29.6 \mathrm{~cm}^{3}$; repair, $67.4 \pm 29.4 \mathrm{~cm}^{3} ; P=.33$; and replacement, $51 \pm 17.9 \mathrm{~cm}^{3}$; repair, $71.5 \pm 40 \mathrm{~cm}^{3} ; P=.1$, respectively). The LV stroke volume was similar in the replacement and repair groups preoperatively $(P=.6)$ and postoperatively $(P=.62)$. The strain, strain rate, and TDI values were all similar between the replacement and repair groups both pre and postoperatively $(P>.05)$. 
TABLE 3. Left ventricular radial strain interrogation

\begin{tabular}{lccr}
\hline \multicolumn{1}{c}{ Variable } & Preoperatively & Postoperatively & $P$ value \\
\hline Radial strain & & & \\
Anteroseptal & $-23.63 \pm 4.66$ & $17.27 \pm 5.97$ & $<.001$ \\
Anterior & $-24.27 \pm 4.98$ & $17.79 \pm 4.18$ & $<.001$ \\
Anterolateral & $-24.54 \pm 4.14$ & $17.63 \pm 5.61$ & $<.001$ \\
Inferolateral & $-25 \pm 4.77$ & $16.7 \pm 6.41$ & $<.001$ \\
Inferior & $-24.21 \pm 10.66$ & $16.94 \pm 7.79$ & $<.001$ \\
Inferoseptal & $-25.88 \pm 4.94$ & $17.42 \pm 9.05$ & $<.001$ \\
4-Chamber view strain & & & \\
Basal septal & $18.27 \pm 4.35$ & $11.69 \pm 5.97$ & $<.001$ \\
Mid septal & $19.15 \pm 5.08$ & $10.93 \pm 5.15$ & $<.001$ \\
Apical septal & $18.83 \pm 5.56$ & $10.75 \pm 6.64$ & $<.001$ \\
Apical lateral & $16.82 \pm 4.44$ & $11.5 \pm 7.95$ & .002 \\
Mid lateral & $15.23 \pm 4.89$ & $11.94 \pm 6.54$ & .009 \\
Basal lateral & $16.35 \pm 4.16$ & $11.55 \pm 7.01$ & .002 \\
\hline
\end{tabular}

Data presented as mean $\pm \mathrm{SD}$

\section{Healthy Volunteers}

The LV EDV was $102.7 \pm 19.2 \mathrm{~cm}^{3}$, the LV ESV was 39.6 $\pm 8.7 \mathrm{~cm}^{3}$, the LV stroke volume was $62.9 \pm 12.5 \mathrm{~cm}^{3}$, and the LVEF was $67.2 \% \pm 4.5 \%$. The longitudinal and radial strain values were similar to the values of the preoperative group $(P>.05)$.

\section{DISCUSSION}

The results of the present study have demonstrated significant improvement of LV geometry and performance after DMR repair, when assessed using 3DE. Furthermore, ST analysis of the 2 populations for deformation demonstrated increased longitudinal strain postoperatively for both groups (repair and replacement). The strain rate was also increased. Within the follow-up period, the effect of chronic $\mathrm{LV}$ volume loading from mitral regurgitation was assumed

TABLE 4. Echocardiographic predictors of mitral regurgitation recurrence after mitral valve repair

\begin{tabular}{llcrc}
\hline & AUC & $\begin{array}{c}\text { Cutoff } \\
\text { value }\end{array}$ & $\begin{array}{c}\text { Sensitivity } \\
(\%)\end{array}$ & $\begin{array}{c}\text { Specificity } \\
(\%)\end{array}$ \\
\hline $\mathrm{A}^{\prime}$ apical septal (cm/s) & 0.823 & $>3.2$ & 100 & 61.5 \\
$\mathrm{~A}^{\prime}$ mid lateral (cm/s) & 0.815 & $\leq 3.66$ & 100 & 61.5 \\
Radial strain mid lateral & $0.814 \leq-27$ & 80 & 78.6 \\
Radial strain apical lateral & 0.8 & $\leq-25$ & 100 & 71.4 \\
TR (mm/s) & 0.743 & $>2.9$ & 100 & 50 \\
S mid lateral (cm/s) & 0.738 & $\leq 4.02$ & 60 & 84.6 \\
MVPPG (mm Hg) & 0.736 & $\leq 94.09$ & 80 & 71.4 \\
MV peak V (cm/s) & 0.736 & $\leq 4.85$ & 80 & 71.4 \\
FS (\%) & 0.714 & $\leq 35$ & 60 & 85.7 \\
A basal septal (cm/s) & 0.708 & $>3.87$ & 100 & 38.5 \\
A mid septal (cm/s) & 0.708 & $>5.14$ & 60 & 76.9 \\
Radial strain rate basal septal & 0.707 & $>1.1$ & 60 & 71.4 \\
Strain mid septal & 0.7 & $\leq 17$ & 80 & 64.3 \\
\hline AUC, Area
\end{tabular}

$A U C$, Area under the curve; $A$, late diastolic wave from tissue Doppler imaging; $S$, systolic wave from tissue Doppler imaging; $T R$, tricuspid regurgitant velocity; $M V P P G$, mitral valve peak pressure gradient; $M V$ peak $V$, mitral wave peak velocity; $F S$, fractional shortening. to lead into reverse remodeling after correction of the pathologic mitral valve.

Analysis of the postoperative group demonstrated a considerable global recovery of the LV function. Within 4 to 6 months after surgery, the LV volumes had decreased significantly (Table 2). The LV EDV demonstrated the greatest change in both groups compared with the LV ESV (comparing the 2 groups pre- and postoperatively). A difference was found between the repair and replacement groups. The LV EDV had improved significantly in the repair group, as demonstrated by the multivariate regression analysis. The stroke volume was decreased postoperatively, with a similar distribution across the 2 groups. The LVEF was similar postoperatively, with a nominal lead in the replacement group. Preoperatively, the left ventricle will have a pseudo-overestimated EF owing to the volume overload. The ventricle after the operation will not have any volume overload; therefore, the LVEF might seem reduced at 6 months compared with the baseline value.

The radial strain was significantly decreased after mitral valve surgery, with an almost equal distribution between the septal and free wall segments and between the 2 groups (Figure 3). This translated to the differential degree of deformation that occurred in the left ventricle (owing to the chronic volume load) and leading to inhomogeneous twists and untwists during the cardiac cycle. From these results, LV torsion appeared to recover within the first 4 to 6 months after mitral valve surgery. Functional homogeneity was also demonstrated from the improvement of the strain rate values across the septal and free wall segments. Although the strain was improved in the left apical septal segment, the overall performance appeared to be better in the basal septal, mid-septal, and basal lateral segments (Figure 3). Longitudinal strain was improved in the apical segment (as was the radial strain); however, the longitudinal strain rate showed significant correction in the mid-septal and basal lateral LV segments. A comparison of the radial and longitudinal strain values concurred with regard to the septal wall (mid and apical), appearing to undergo recovery after mitral valve surgery, followed by the basal lateral free wall.

Furthermore, it was found that reduced late diastolic waves of the apical and mid-septal walls can predict recurrent mitral regurgitation after surgery. The reduced velocity of late diastolic wave represents severe diastolic dysfunction owing to reduced ventricular relaxation. The clinical importance of our finding has confirmed the role of diastolic dysfunction as an early predictor of clinical outcome. ${ }^{18}$ ST can identify the underlying diastolic dysfunction in degenerative mitral valve disease, which could be masked by good systolic function of the hyperdynamic left ventricle.

\section{Implications of Study Findings in Clinical Practice}

The clinical implication of these observations is that symptom and cardiac function improvement are present shortly after surgical intervention. This has a direct effect on the 
outcome after mitral valve surgery and the patient's life expectancy and survival. Also, the ability of these novel echocardiographic methods to detect subtle changes in the myocardial function at an early disease stage offers room for thought, decision, and management. Enriquez-Sarano and colleagues ${ }^{19}$ have stressed the importance of quantitative assessment of mitral regurgitation, because the term "watchful waiting" has been questioned. ${ }^{20}$ Surgery of patients with advanced disease and higher functional NYHA class has increased risk and mortality.

\section{Echocardiography Predictors of Mitral Valve Repair}

Recurrence of mitral regurgitation after mitral valve repair remains a cause of concern. To date, only operative complications, preoperative patient risk factors/comorbidities, and disease progression have been identified as the causes of disease recurrence. In the present study, we attempted to identify preoperative factors pertaining to the $\mathrm{LV}$ dimensions and performance, which might be present in subtle subclinical form, not allowing for symptomatic concern and usually missed by contemporary $2 \mathrm{DE}$. The LV functional status can be grossly quantified using the latter according to the LVEF, visible segmental hypokinesis, and enlarged diameters. In contrast, 3DE and ST were able to identify early changes in the LV performance, ${ }^{21,22}$ in particular, regional diastolic dysfunction, which can point the physician to the affected segments, allowing for a comprehensive opinion on the optimal timing for surgical referral. It is also evident that new postoperative changes in the regional diastolic dysfunction can be identified and related to the surgical technique used (e.g., a suboptimal length of artificial chordae or alteration of the annular geometry, which can compromise the performance of repair during follow-up). ${ }^{23}$

\section{Confounding Factors}

Prolapsing or flailing leaflets were assessed according to standard criteria and Carpentier's functional classification with a precise characterization of the involved scallops or segments. The long-term success and the incidence of recurrent mitral regurgitation, however, will be determined by several confounding anatomic and physiologic factors related to the left atrium, leaflets, mitral annulus, chordae, papillary muscles, and left ventricle. It is also important to consider the different selection of the size of the mitral valve ring and the repair/replacement technique. The confounding factors might have been a significant limitation of our study, because the sample size of our patients was small and the subanalysis would not be accurate; therefore, such factors have not been assessed in detail.

\section{Limitations and Future Research}

A larger patient cohort might have improved the power of the present study and would validate these results. A comparison of the findings among different centers will add useful in- formation pertaining to the reproducibility and interoperator bias. It would be equally important to analyze the role and changes incurred in the right ventricle during chronic volume loading conditions and after mitral valve surgery, as well as the role of concomitant tricuspid repair.

A low mitral valve peak pressure gradient was paradoxically associated with a greater risk of mitral valve repair failure. However, a number of limitations exist. The mitral valve peak velocity is influenced by the left ventricle and left atrial pressure, as well as contractility.

Owing to the small number of patients with differing ring diameters, a limitation of the study was that we did not perform a separate analysis for the effect on the implanted valve or ring type on the results. However, the criteria for implantation were equal for all patients.

\section{CONCLUSIONS}

The results highlighted that in certain cases, despite a flawless surgical technique, and demonstrated by an intraoperative transesophageal echocardiography after exiting from the bypass, the repaired valve failed to sustain its newly reacquired capacity to withstand regurgitation, with recurrence evident within 6 months after surgery. 3DE and ST proved to be important imaging tools in assessing reverse LV remodeling after DMR surgery. Subtle regional preoperative changes in diastolic function of the septal and lateral wall might be identified preoperatively, aiding in optimizing the referral timing and recognizing potential culprits as indicators of disease recurrence after mitral repair. Additional research is required on the use of complete or rigid annuloplasty rings to assess whether these might cause additional hindering of LV function.

\section{References}

1. Hunter JJ, Chien KR. Signaling pathways for cardiac hypertrophy and failure. $N$ Engl J Med. 1999;341:1276-83.

2. Ross J Jr. Adaptations of the left ventricle to chronic volume overload. Circ Res. 1974;34:II-64-70.

3. Spinale FG, Ishihra K, Zile M, DeFryte G, Crawford FA, Carabello BA. Structural basis for changes in left ventricular function and geometry because of chronic mitral regurgitation and after correction of volume overload. J Thorac Cardiovasc Surg. 1993;106:1147-57.

4. Bonow RO, Carabello BA, Chatterjee K, de Leon AC Jr, Faxon DP, Freed MD, et al. ACC/AHA 2006 guidelines for the management of patients with valvular heart disease: a report of the American College of Cardiology/American Heart Association Task Force on Practice Guidelines. JACC. 2006;48:e1-148.

5. Bonow RO, Carabello BA, Chatterjee K, de Leon AC Jr, Faxon DP, Freed MD, et al. 2008 Focused update incorporated into the ACC/AHA 2006 Guidelines for the Management of Patients with Valvular Heart Disease. Circulation. 2008;118: e523-661.

6. Lang RM, Mor-Avi V, Sugeng L, Nieman PS, Sahn DJ. Three-dimensional echocardiography, the benefits of the additional dimension. Am J Cardiol. 2006;48: 2053-69.

7. Zoghbi WA, Enriquez-Sarano M, Foster E, Grayburn PA, Kraft CD, Levine RA, et al. Recommendations for evaluation of the severity of native valvular regurgitation with two-dimensional and Doppler echocardiography. J Am Soc Echocardiogr. 2003;16:777-802.

8. Maffessanti F, Nesser HJ, Weinert L, Steringer-Mascherbauer R, Niel J, Gorissen W, et al. Quantitative evaluation of regional left ventricular function 
using three-dimensional speckle tracking echocardiography in patients with and without heart disease. Am J Cardiol. 2009;104:1755-62.

9. Hung J, Lang RM, Flachskampf FA, Shernan SK, McCulloch ML, Adams DB, et al. 3D echocardiography: a review of the current status and future directions. J Am Soc Echocardiogr. 2007;20:213-33.

10. Maffessanti F, Caiani EG, Tamborini G, Muratori M, Sugeng L, Weinert L, et al. Serial changes in left ventricular shape following early mitral valve repair. Am J Cardiol. 2010;106:836-42.

11. Pislaru C, Abraham TP, Belohlavek M. Strain and strain rate echocardiography. Curr Opin Cardiol. 2002; 17:443-54.

12. Van Dalen BM. Feasibility and reproducibility of left ventricular rotation parameters measured by speckle tracking echocardiography. Eur J Echocardiogr. 2009; 10:669-76.

13. de Agustin JA, Pérez de Isla L, Núñez-Gil IJ, Vivas D, Manzano Mdel C, MarcosAlberca P, et al. Assessment of myocardial deformation: predicting medium-term left ventricular dysfunction after surgery in patients with chronic mitral regurgitation. Rev Esp Cardiol. 2010;63:544-53.

14. Adams DH, Rosenhek R, Falk V. Degenerative mitral valve regurgitation: best practice revolution. Eur Heart J. Epub ahead of print 11 July 2010.

15. Kuppahally SS, Akoum N, Burgon NS, Badger TJ, Kholmovski EG, Vijayakumar S, et al. Left atrial strain and strain rate in patients with paroxysmal and persistent atrial fibrillation: relationship to left atrial structural remodeling detected by delayed-enhancement MRI. Circ Cardiovasc Imaging. 2010;3:231-9.
16. Grapsa J, O'Regan DP, Pavlopoulos H, Durighel G, Dawson D, Nihoyannopoulos P. Right ventricular remodeling in pulmonary arterial hypertension with three-dimensional echocardiography: comparison with cardiac magnetic resonance. Eur J Echocardiogr. 2010;11:64-73.

17. Hamley JA. Receiver operating characteristic (ROC) methodology: the state of the art. Crit Rev Diagn Imaging. 1989;29:307-35.

18. Wachtell K, Palmieri V, Gerdts E, Bella JN, Aurigemma GP, Papademetriou V, et al. Prognostic significance of left ventricular diastolic dysfunction in patients with left ventricular hypertrophy and systemic hypertension (the LIFE Study). Am J Cardiol. 2010;106:999-1005.

19. Enriquez-Sarano M, Akins CW, Vahanian A. Mitral regurgitation. Lancet. 2009; 373:1382-94.

20. Rosenhek R, Rader F, Klar U, Gabriel H, Krejc M, Kalbeck D, et al. Outcome of watchful waiting in asymptomatic severe mitral regurgitation. Circulation. 2006; 18:2238-44.

21. Nesbitt G, Mankad S, Oh J. Strain imaging in echocardiography: methods and clinical applications. Int J Cardiovasc Imaging. 2009;25: 9-22.

22. Greenbaum RA, Ho SY, Gibson DG, Becker AE, Anderson RH. Left ventricular fibre architecture in man. Br Heart J. 1981;45:248-63.

23. Zegdi R, Sleilaty G, Latremouille C, Berrebi A, Carpentier A, Deloche A, et al Reoperation for failure of mitral valve repair in degenerative disease: a singlecenter experience. Ann Thorac Surg. 2008;86:1480-4. 